\title{
Sparse data embedding and prediction by tropical matrix factorization
}

\author{
Amra Omanović ${ }^{1}$, Hilal Kazan², Polona Oblak ${ }^{1}$ and Tomaž Curk*
}

\author{
${ }^{*}$ Correspondence: \\ tomaz.curk@fri.uni-lj.si \\ ${ }^{1}$ Faculty of Computer \\ and Information Science, \\ University of Ljubljana, Večna \\ pot 113, 1000 Ljubljana, \\ Slovenia \\ Full list of author information \\ is available at the end of the \\ article
}

\begin{abstract}
Background: Matrix factorization methods are linear models, with limited capability to model complex relations. In our work, we use tropical semiring to introduce nonlinearity into matrix factorization models. We propose a method called Sparse Tropical Matrix Factorization (STMF) for the estimation of missing (unknown) values in sparse data.

Results: We evaluate the efficiency of the STMF method on both synthetic data and biological data in the form of gene expression measurements downloaded from The Cancer Genome Atlas (TCGA) database. Tests on unique synthetic data showed that STMF approximation achieves a higher correlation than non-negative matrix factorization (NMF), which is unable to recover patterns effectively. On real data, STMF outperforms NMF on six out of nine gene expression datasets. While NMF assumes normal distribution and tends toward the mean value, STMF can better fit to extreme values and distributions.
\end{abstract}

Conclusion: STMF is the first work that uses tropical semiring on sparse data. We show that in certain cases semirings are useful because they consider the structure, which is different and simpler to understand than it is with standard linear algebra.

Keywords: Data embedding, Matrix factorization, Tropical factorization, Sparse data, Matrix completion, Tropical semiring

\section{Background}

Matrix factorization methods are getting increasingly popular in many research areas [1-3]. These methods generate linear models, which cannot model complex relationships. Our work focuses on incorporating non-linearity into matrix factorization models by using tropical semiring.

The motivation for using tropical matrix factorization can be seen in the classic example of movie rating data, where a users-by-movies matrix contains the rating users assigned to movies. In standard matrix factorization methods, it is assumed that a user's final rating is a linear combination of some factors (a person likes some movie because of the director, the genre, the lead actor, etc.). But it is also possible that some factor is so dominant that all others are irrelevant. An example given for the Latitude algorithm author(s) and the source, provide a link to the Creative Commons licence, and indicate if changes were made. The images or other third party material in this article are included in the article's Creative Commons licence, unless indicated otherwise in a credit line to the material. If material is not included in the article's Creative Commons licence and your intended use is not permitted by statutory regulation or exceeds the permitted use, you will need to obtain permission directly from the copyright holder. To view a copy of this licence, visit http:// creativecommons.org/licenses/by/4.0/. The Creative Commons Public Domain Dedication waiver (http://creativecommons.org/publi cdomain/zero/1.0/) applies to the data made available in this article, unless otherwise stated in a credit line to the data. 
[4], a person likes all Star Wars movies irrespective of actors or directors, shows that using the max operator instead of the sum might produce a better model.

We develop a method for the prediction of missing (unknown) values, called Sparse Tropical Matrix Factorization (STMF). We evaluate its performance on the prediction of gene expression measurements from The Cancer Genome Atlas Research Network (TCGA) database. We show that the newly defined operations can discover patterns, which cannot be found with standard linear algebra.

\section{Related work}

Matrix factorization is a data embedding model which gives us a more compact representation of the data and simultaneously finds a latent structure. The most popular example is the non-negative matrix factorization (NMF) [5], where the factorization is restricted to the matrices with non-negative entries. This non-negativity in resulting factor matrices makes the results easier to interpret. One of the applications of matrix factorization methods is for recommender systems, where users and items are represented in a lower-dimensional latent space [6]. Binary matrix factorization $(\mathrm{BMF})[7,8]$ is a variant rooted from NMF where factor matrices are binary, while probabilistic non-negative matrix factorization (PMF) [9] models the data as a multinomial distribution. MMDNMF [10] is a supervised NMF method, which minimizes the maximum distance within-class and maximizes the minimum distance between-class. Integrative approaches, which use standard linear algebra to simultaneously factorize multiple data sources and improve predictive accuracy, are reviewed in [11]. Multi-omic and multi-view clustering methods like MultiNMF [12], Joint NMF [13], PVC [14], DFMF [15], MDNMF [16] and iONMF [17] can be used for data fusion of multiple data sources.

Lately, subtropical semiring $(\max , \cdot)$ gained interest in the field of machine learning, since it can discover interesting patterns $[18,19]$. By taking the logarithm of the subtropical semiring, we obtain the tropical semiring $(\max ,+)[20]$. Although these two semirings are isomorphic, the factorization in tropical semiring works differently than the factorization in subtropical semiring. The Cancer algorithm [20] works with continuous data, performing subtropical matrix factorization $(\mathrm{SMF})$ on the input matrix. Two main components of the algorithm are: iteratively updating the rank-1 factors one-byone and approximate the max-times reconstruction error with a low-degree polynomial. Latitude algorithm [4] combines NMF and SMF, where factors are interpreted as NMF features, SMF features or as mixtures of both. This approach gives good results in cases where the underlying data generation process is a mixture of the two processes. In [21] authors used subtropical semiring as part of a recommender system. We can consider their method to be a particular kind of neural network. Le Van et al. [22] presented a single generic framework that is based on the concept of semiring matrix factorization. They applied the framework on two tasks: sparse rank matrix factorization and rank matrix tiling.

De Schutter \& De Moor [23] presented a heuristic algorithm to compute factorization of a matrix in the tropical semiring, which we denote as Tropical Matrix Factorization (TMF). They use it to determine the minimal system order of a discrete event system (DES). In the last decades, there has been an increase of interest in this research area, and DES is modeled as a max-plus-linear (MPL) system [24, 25]. 
In contrast to TMF where approximation error is reduced gradually, convergence is not guaranteed in the Cancer algorithm. Both Cancer and TMF return factors that encode the most dominant components in the data. However, by their construction, they cannot be used for prediction tasks in different problem domains, such as predicting gene expression. In contrast with the NMF method and its variants, which require non-negative data, TMF can work with negative values.

Hook [26] reviewed algorithms and applications of linear regression over the maxplus semiring, while Gärtner and Jaggi [27] constructed a tropical analogue of support vector machines (SVM), which can be used to classify data into more than just two classes compared to the classical SVM. Zhang et al. [28] in their work establish a connection between neural networks and tropical geometry. They showed that linear regions of feedforward neural networks with rectified linear unit activation correspond to vertices of polytopes associated with tropical rational functions. Therefore, to understand specific neural networks, we need to understand relevant tropical geometry. Since one goal in biology is not just to model the data, but also to understand the underlying mechanisms, the matrix factorization methods can give us a more straightforward interpretation than neural networks. The GCN-MF framework [29] uses matrix factorization to combine embeddings and Graph Convolutional Network (GCN) using standard linear algebra. The authors state that matrix factorization only utilizes the linear relationship between entities. When data is more complex, the matrix factorization method cannot identify non-linear relationships. Since deep learning uses non-linear functions and layer combinations, neural networks can learn more complex data patterns. In our work, instead of introducing deep learning, we address the issue of non-linearity with tropical semiring.

In our work, we answer the question stated in Cancer: can tropical factorization be used, in addition to data analysis, also in other data mining and machine learning tasks, e.g. matrix completion? We propose a method STMF, which is based on $\mathrm{TMF}$, and it can simultaneously predict missing values, i.e. perform matrix completion. In Table 1 we compare the most relevant methods for our work. To the best of our knowledge STMF is the only method which performs prediction tasks in tropical semiring. STMF introduces non-linearity into matrix factorization models, which enables discovering the most dominant patterns, leading to a more straightforward visual interpretation compared to other methods for missing value prediction.

Table 1 A comparison between different matrix factorization methods

\begin{tabular}{lllll}
\hline & Arithmetic & Data sources & $\begin{array}{l}\text { Prediction } \\
\text { tasks }\end{array}$ & Convergence \\
\hline NMF [5], BMF [7], PMF [9], MMDNMF [10] & Standard & Single & Yes & Yes \\
DFMF [15], i ONMF [17], MDNMF [16] & Standard & Multiple & Yes & Yes \\
Latitude [4] & Standard \& Subtropical & Single & No & No \\
Cancer [20] & Subtropical & Single & No & No \\
TMF [23] & Tropical & Single & No & Yes \\
STMF & Tropical & Single & Yes & Yes \\
\hline
\end{tabular}




\section{Methods}

\section{Tropical semiring and factorization}

Now, we give some formal definitions regarding the tropical semiring. The $(\max ,+)$ semiring or tropical semiring $\mathbb{R}_{\max }$, is the set $\mathbb{R} \cup\{-\infty\}$, equipped with max as addition $(\oplus)$, and + as multiplication $(\otimes)$. For example, $2 \oplus 3=3$ and $1 \otimes 1=2$. On the other hand, in the subtropical semiring or $(\max , \times)$ semiring, defined on the same set $\mathbb{R} \cup\{-\infty\}$, addition ( $\max$ ) is defined as in the tropical semiring, but the multiplication is the standard multiplication $(\times)$. Throughout the paper, symbols + and - refer to standard operations of addition and subtraction. Tropical semiring can be used for optimal control [30], asymptotics [31], discrete event systems [32] or solving a decision problem [33]. Another example is the well-known game Tetris, which can be linearized using the $(\max ,+)$ semiring [34].

Let $\mathbb{R}_{\max }^{m \times n}$ define the set of all $m \times n$ matrices over tropical semiring. For $A \in \mathbb{R}_{\max }^{m \times n}$ we denote by $A_{i j}$ the entry in the $i$-th row and the $j$-th column of matrix $A$. We denote the sum of matrices $A, B \in \mathbb{R}_{\max }^{m \times n}$ as $A \oplus B \in \mathbb{R}_{\max }^{m \times n}$ and define its entries as

$$
(A \oplus B)_{i j}=A_{i j} \oplus B_{i j}=\max \left\{A_{i j}, B_{i j}\right\},
$$

$i=1, \ldots, m, j=1, \ldots, n$. The product of matrices $A \in \mathbb{R}_{\max }^{m \times p}, B \in \mathbb{R}_{\max }^{p \times n}$ is denoted by $A \otimes B \in \mathbb{R}_{\max }^{m \times n}$ and its entries are defined as

$$
(A \otimes B)_{i j}=\bigoplus_{k=1}^{p} A_{i k} \otimes B_{k j}=\max _{1 \leq k \leq p}\left\{A_{i k}+B_{k j}\right\}
$$

$i=1, \ldots, m, j=1, \ldots, n$.

Matrix factorization over a tropical semiring is a decomposition of a form $R=U \otimes V$, where $R \in \mathbb{R}_{\max }^{m \times n}, U \in \mathbb{R}_{\max }^{m \times r}, V \in \mathbb{R}_{\max }^{r \times n}$ and $r \in \mathbb{N}_{0}$. Since for small values of $r$ such decomposition may not exist, we state tropical matrix factorization problem as: given a matrix $R$ and factorization rank $r$, find matrices $U$ and $V$ such that

$$
R \cong U \otimes V
$$

To implement a tropical matrix factorization algorithm, we need to know how to solve tropical linear systems. Methods for solving linear systems over tropical semiring differ substantially from methods that use standard linear algebra [34].

We define the ordering in tropical semiring as $z \preceq w$ if and only if $z \oplus w=w$ for $z, w \in \mathbb{R}_{\max }$, and it induces the ordering on vectors and matrices over tropical semiring entry-wise. For $A \in \mathbb{R}_{\max }^{m \times n}$ and $c=\left[c_{k}\right] \in \mathbb{R}_{\max }^{m}$ the system of linear inequalities $A \otimes x \preceq c$ always has solutions and we call the solutions of $A \otimes x \preceq c$ the subsolutions of the linear system $A \otimes x=c$. The greatest subsolution $x=\left[x_{1} x_{2} \ldots x_{n}\right]^{T}$ of $A x=c$ can be computed by

$$
x_{i}=\min _{1 \leq j \leq m}\left(c_{j}-A_{j i}\right)
$$

for $i=1,2, \ldots, n$. We will use (2) in a column-wise form to solve the matrix equations.

TMF starts with an initial guess for the matrix $U$ in (1), denoted by $U_{0}$ and then computes $V$ as the greatest subsolution of $U_{0} \otimes X=R$. Then authors use the iterative procedure by selecting and adapting an entry of $U$ or $V$ and recomputing it as the greatest subsolution of 
$Y \otimes V=R$ and $U \otimes X=R$, respectively. The $b$-norm of matrix $W$, defined as the objective function $\|W\|_{b}=\sum_{i, j}\left|W_{i j}\right|$ is used to minimize the approximation error $\|R-U \otimes V\|_{b}$.

\section{Our contribution}

In our work, we implement and modify TMF so that it can be applied in data mining tasks. We propose a sparse version of TMF, which can work with missing values.

In Sparse Tropical Matrix Factorization (STMF), which is available on https://github.com/ Ejmric/STMF, we update the factor matrices $U$ and $V$ based on the selected given entry of the input data matrix $R$ to predict the missing values in $R$. In Algorithm 1, we present the pseudocode of STMF in which for each given entry $(i, j)$ of $R$ we first update $U$ and $V$ based on the element from the $i$ th row of the left factor $U$ (ULF, see Algorithm 2). If the update of the factors does not improve the approximation of $R$, then we update $U$ and $V$ based on the element from the $j$ th column of the right factor $V$ (URF, see Algorithm 3).

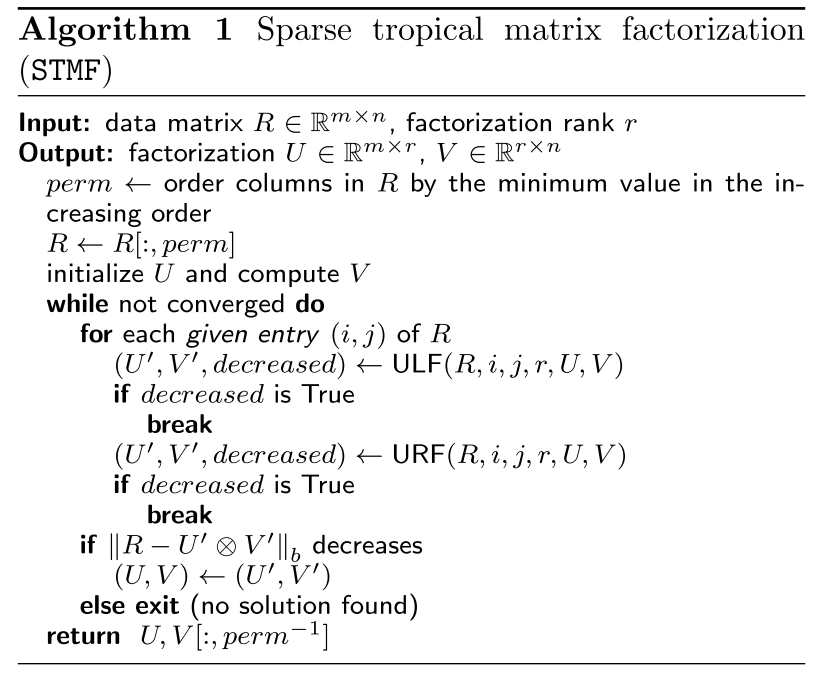

Algorithms ULF and URF differ from the corresponding TMF's versions in the way they solve linear systems. Since some of the entries of matrix $A$ are not given, we define $(\mathrm{min},+)$ matrix multiplication $\otimes^{*}$ as

$$
\left(A \otimes \otimes^{*} B\right)_{i j}=\min _{A_{i k}, B_{k j} \text { are given }}\left\{A_{i k}+B_{k j}\right\}
$$

for matrices $A \in \mathbb{R}_{\max }^{m \times p}$ and $B \in \mathbb{R}_{\max }^{p \times n}, i=1, \ldots, m, j=1, \ldots, n$. Newly-defined operator $\otimes^{*}$ can be seen as a generalization of Eq. (2), and it is used for solving linear systems by skipping unknown values. We assume that at least one element in each row/column is known. 

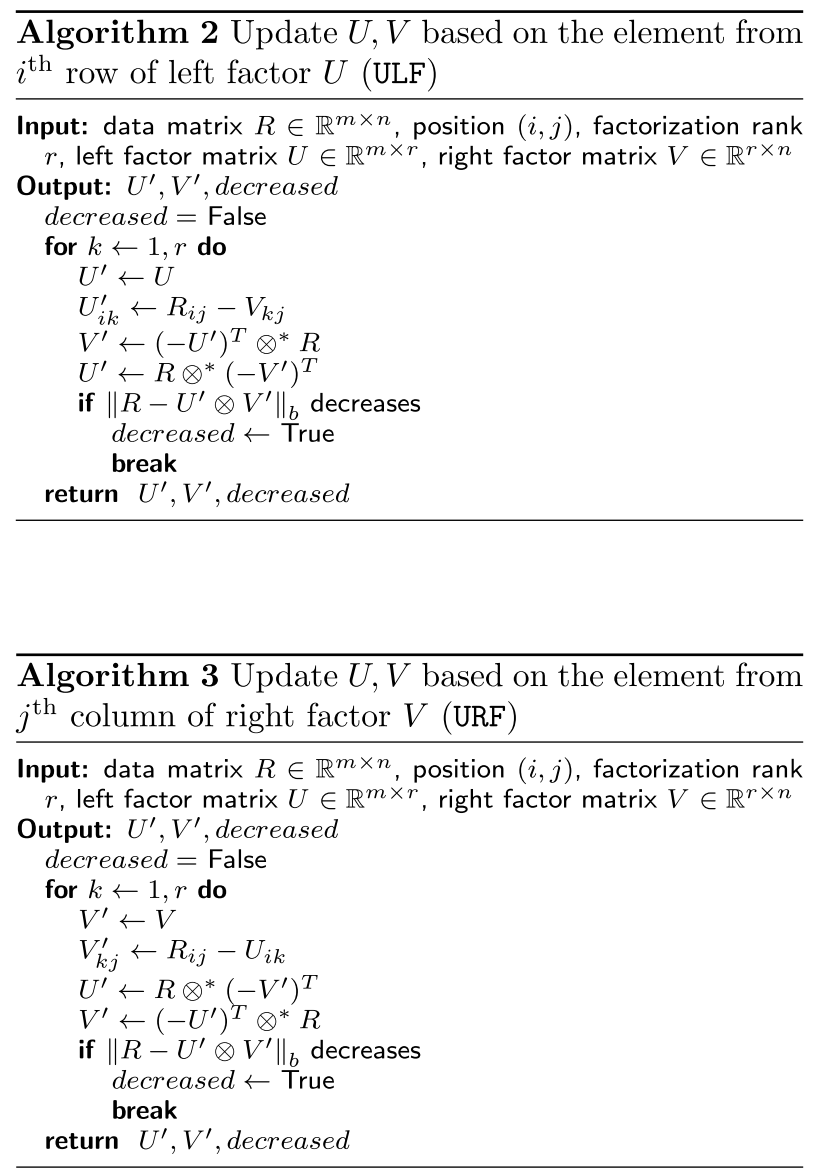

Among the different matrix initialization strategies, we obtained the best performance with Random Acol strategy [15, 35]. Random Acol computes each column of the initialized matrix $U$ as an element-wise average of a random subset of columns of the data matrix $R$. It is a widely used method for initializations in matrix factorization methods since it gives better insight into the original data matrix than simple random initialization.

In contrast to Cancer, where convergence is not guaranteed, the update rules of STMF, similar to TMF, gradually reduce the approximation error. This is ensured by the fact that factor matrices $U$ and $V$ are only updated in the case when $\|R-U \otimes V\|_{b}$ monotonously decreases.

\section{Distance correlation}

It is well known that Pearson and Spearman correlation coefficients can misrepresent non-linear relationships [36]. Since in real data, we often deal with non-linearity, our choice is to use so-called distance correlation. Distance correlation [37] is a straightforward measure of association that uses the distances between observations as part of its calculation. It is a better alternative for detecting a wide range of relationships between variables. 
Let $X$ and $Y$ be the matrices each with $n$ rows and $A$ and $B$ their matrices of Euclidean distances with the row/column means subtracted, and grand mean added. After matrix centering the distance covariance $V_{x y}$ is defined as

$$
V_{X Y}^{2}=\frac{1}{n^{2}} \sum_{i, j=1}^{n} A_{i j} B_{i j},
$$

and distance correlation dcor as

$$
\operatorname{dcor}(X, Y)=\sqrt{\frac{V_{X Y}^{2}}{V_{X} V_{Y}}},
$$

where $V_{X}$ and $V_{Y}$ represent distance variances of matrices $X$ and $Y$. Distance correlation is 0 only if the two corresponding variables are independent.

Distance correlation cannot be used to compare specific rows between $X$ and $Y$, because it requires the entire matrix to be centered first. In such cases we use Euclidean norm between rows of centered original and rows of centered approximated data.

\section{Synthetic data}

We create two types of synthetic datasets of rank 3: one smaller of size $200 \times 100$ and five larger of size $500 \times 300$. We use the $(\max ,+)$ multiplication of two random nonnegative matrices sampled from a uniform distribution over $[0,1)$ to generate each synthetic dataset.

\section{Real data}

We download the preprocessed TCGA data [11] for nine cancer types, where for each cancer type three types of omic data are present: gene expression, methylation and miRNA data. We transpose the data sources, so that in each data source, the rows represent patients and columns represent features. The first step of data preprocessing is to take the subset of patients for which we have all three data sources. In our experiments we use only gene expression data. After filtering the patients, we substitute each gene expression value $x$ in the original data with the $\log _{2}(x+1)$. With log-transformation, we make the gene expression data conform more closely to the normal distribution, and by adding one, we reduce the bias of zeros. We also perform polo clustering, which is an optimal linear leaf ordering [38], to re-order rows and columns on the preprocessed data matrix. Polo clustering results in a more interpretative visualization of factor matrices.

Next, we use feature agglomeration to merge similar genes by performing clustering [39]. We use Ward linkage and split genes into 100 clusters (see Additional file 1: Figure S 24), the center of each cluster representing a meta-gene. With this approach, we minimize the influence of non-informative, low variance genes on distance calculations and reduce the computational requirements.

For Breast Invasive Carcinoma (BIC), we do not perform feature agglomeration since a list of 50 genes, called PAM50 [40], classify breast cancers into one of five subtypes: LumA, LumB, Basal, Her2, and Normal [41, 42], resulting in our BIC data matrix of size $541 \times 50$. These five subtypes differ significantly in the expression of only a few genes in 
BIC data, which leads to the value close to zero for silhouette score [43] (see Additional file 1: Figure S 22). The sizes of the final nine datasets are listed in Table 2.

\section{Performance evaluation}

Since STMF is the first work in tropical semiring, which performs matrix completion, we choose NMF as a baseline method because it represents the original matrix factorization method using standard linear algebra. In contrast, other methods from Table 1, which use standard linear algebra, are extensions of NMF. Additionally, we provide running time (Table 3) and distance correlation (Table 4) results for PMF because it represents the advanced version of NMF that is suitable for performing prediction task on a single dataset.

Experiments were performed for varying values of the factorization rank. The smaller synthetic dataset experiments were run 10 times, with 500 iterations each, and on larger synthetic datasets, experiments were run 50 times, with 500 iterations each. Experiments for real data were run five times, with 500 iterations. For both datasets, we mask randomly and uniformly $20 \%$ of data as missing, which we then use as a test set to evaluate the tested methods. We assume that in a typical dataset, data will be missing uniformly at random. The remaining $80 \%$ represent the training set. We choose a rank based on the approximation error on training data, which represents a fair/optimal choice for both methods, STMF and NMF so that we can compare them, knowing both of them to have the same number of parameters.

We compute the distance correlation and Euclidean norm between the original and approximated data matrix to evaluate the predictive performance.

\section{Results}

First, we use synthetic data to show the correctness of the STMF algorithm. We use the smaller dataset to show that STMF can discover the tropical structure. The larger datasets are needed to show how the order of rows and columns affects the result. We then apply it to real data to compare the performance and interpretability of models obtained with STMF and NMF.

Table 2 Size of gene expression data in the form of patients $\times$ meta-genes for eight cancer subtypes, and for the subset of PAM50 genes in BIC

\begin{tabular}{ll}
\hline Cancer subtype & Size \\
\hline Acute Myeloid Leukemia (AML) & $171 \times 100$ \\
Colon Adenocarcinoma (COLON) & $221 \times 100$ \\
Glioblastoma Multiforme (GBM) & $274 \times 100$ \\
Liver Hepatocellular Carcinoma (LIHC) & $410 \times 100$ \\
Lung Squamous Cell Carcinoma (LUSC) & $344 \times 100$ \\
Ovarian serous Cystadenocarcinoma (OV) & $291 \times 100$ \\
Skim Cutaneous Melanoma (SKCM) & $450 \times 100$ \\
Sarcoma (SARC) & $261 \times 100$ \\
Breast Invasive Carcinoma (BIC) & $541 \times 50$ \\
\hline
\end{tabular}



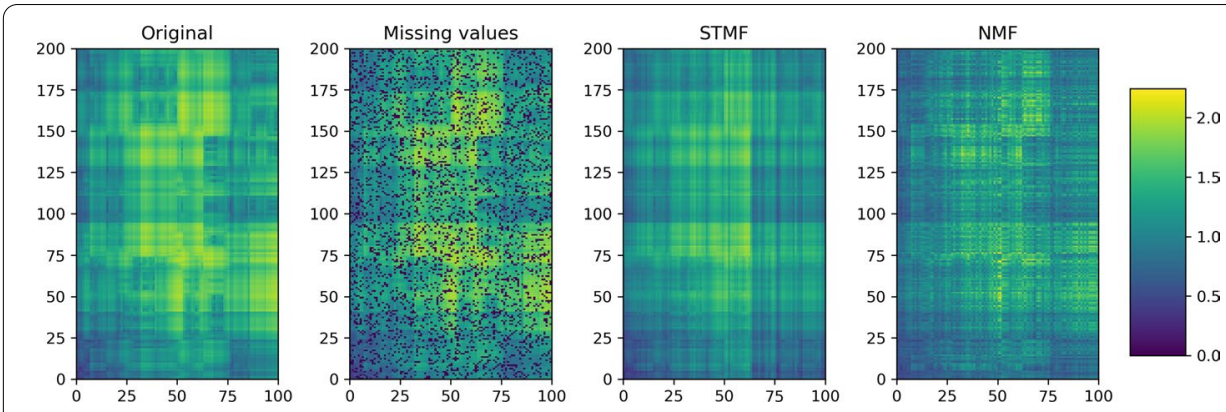

Fig. 1 A comparison between STMF's and NMF's predictions of best rank 4 approximations on $200 \times 100$ synthetic $(\max ,+)$ matrix with $20 \%$ missing values

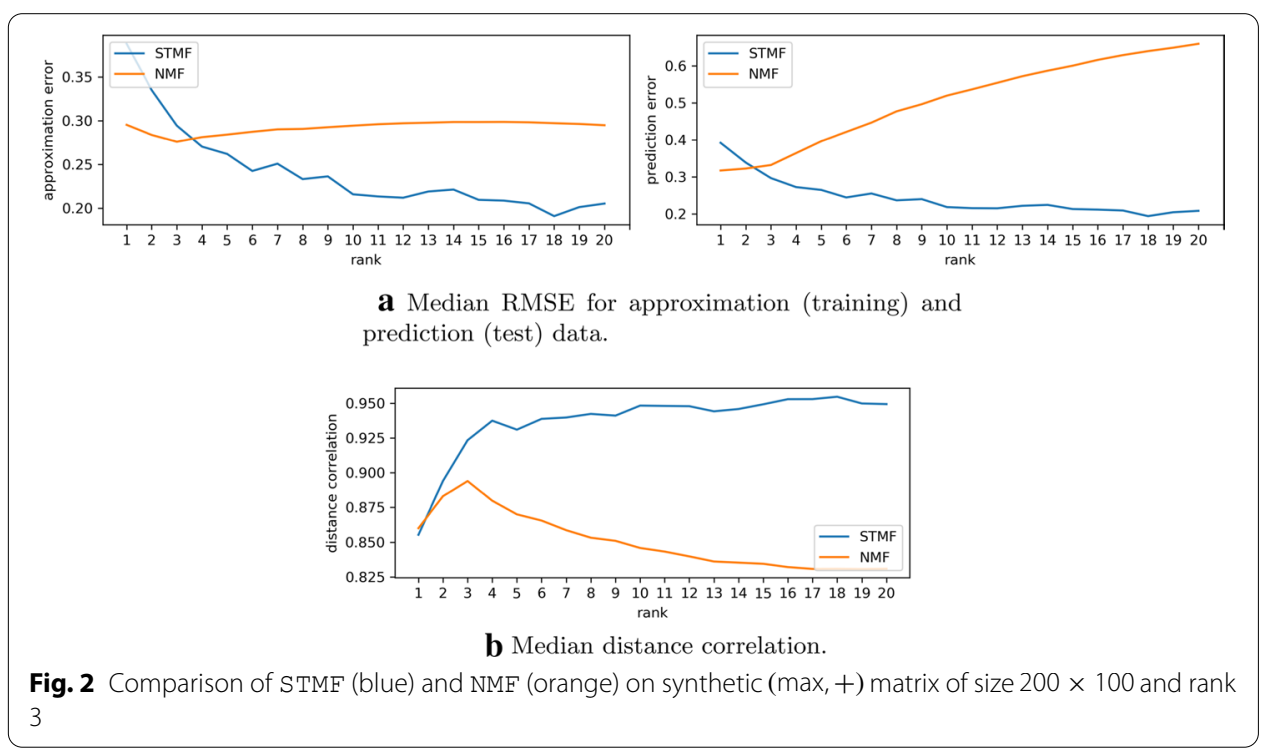

\section{Synthetic data}

The objective of synthetic experiments is to show that STMF can identify the $(\max ,+)$ structure when it exists. Even on a relatively small $200 \times 100$ matrix results show that NMF cannot successfully recover extreme values compared to STMF, see Fig. 1. NMF and $\mathrm{PMF}$ tend towards zero values, which results in a blurry visualization of the approximation matrices (Additional file 1, Subsection 1.1.1). The values of the matrices predicted by NMF and PMF that arise from missing values are much smaller that the values on the same positions in the matrix predicted by STMF. This implies that STMF is more efficient when predicting extreme values. This effect is even more pronounced when the missing values are not missing at random (Additional file 1, Subsection 1.1.2), supporting previous reports by Lin and Boutros [44]. STMF demonstrates to be more robust to the choice of sampling strategy of missing values. As the results show STMF achieves a smaller prediction root-mean-square-error (RMSE) and higher distance correlation (Fig. 2).

Experiments on synthetic data show that changing the execution order of URF and ULF in the computation of STMF does not affect the result of the algorithm.

The result of STMF depends on the order of matrix entries. We perform different types of permutation techniques to order columns and rows on five large synthetic datasets 


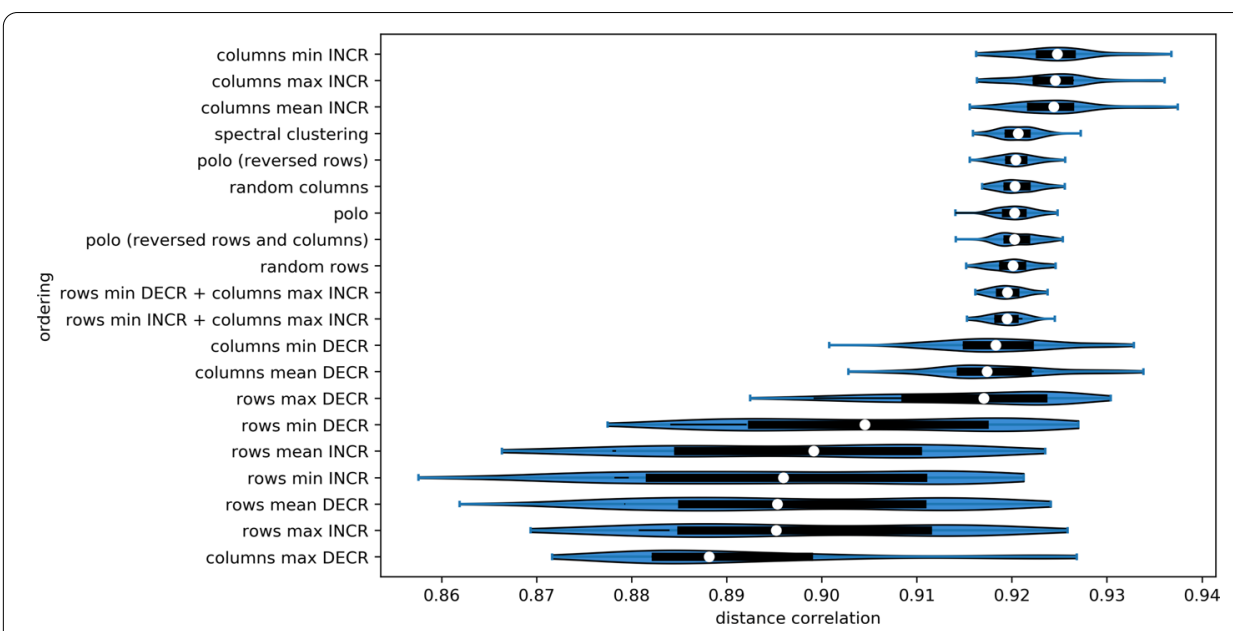

Fig. 3 Effect of ordering strategy on achieved distance correlation by STMF, on $500 \times 300$ synthetic $(\max ,+)$ matrix. Top three performing strategies order columns by increasing values

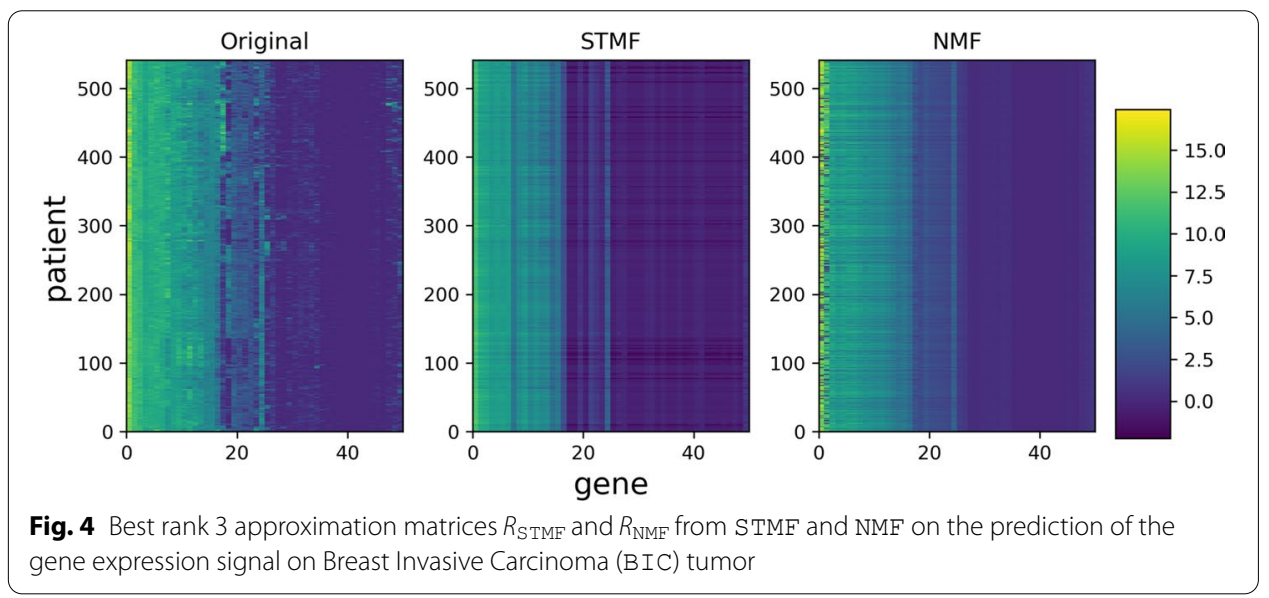

(see Additional file 1: Figure S 19). Top three strategies are to sort columns by increasing values of their minimum, maximum, and mean value (Fig. 3). Moreover, in four out of five datasets, the best results were obtained by ordering columns in increasing order by their minimum value (see Additional file 1: Figure $S$ 20). This strategy represents the first step of STMF method (Algorithm 1).

\section{Real data}

Figure 4 shows the results on BIC matrix, with PAM50 genes and 541 patients. Our findings confirm that STMF expresses some extreme values. We see that STMF successfully recovers large values, while NMF has the largest error where gene expression values are high. Note that NMF tends towards the mean value. Half of the original data is close to zero (plotted in dark blue), which is a reason that NMF cannot successfully predict high (yellow) values. For all other datasets approximation matrices are available in Additional file 1 , Section 2. 
In Fig. 5a we see that NMF has smaller approximation error than STMF, but larger prediction error. So, NMF better approximates/fits the data, but STMF is not prone to overfitting, since its prediction error is smaller. On the other hand, in Fig. 5b, STMF has better distance correlation and silhouette score values; silhouette score for PMF is shown in Additional file 1: Figure S 23. Thus, STMF can find clusters of patients with the same subtype better than NMF, which tends to describe every patient by the mean values in data. For all other datasets similar graphs are available in Additional file 1, Section 2.

The rank three factor matrices of the BIC matrix (see Fig. 4) are illustrated in Fig. 6, where we denote STMF's factor matrices by $U_{\mathrm{STMF}}, V_{\mathrm{STMF}}$, and NMF's factor matrices by $U_{\mathrm{NMF}}, V_{\mathrm{NMF}}$. We see that these factor matrices are substantially different. Basis factor $V_{\mathrm{STMF}}$ (first and third row) is visually the most similar to the original matrix than any other factor alone. Factor $V_{\text {STMF }}$ detects low and high values of gene expression, while factor $V_{\mathrm{NMF}}$ detects high values in the first two columns (second and third row, respectively) and low values in remaining columns (first row). Coefficient factors $U_{\mathrm{STMF}}$ and $U_{\mathrm{NMF}}$ contribute to a good approximation of the original matrix. For all other datasets factor matrices are available in Additional file 1, Section 2.

To see which part of data is explained by which factorization rank, we define a latent matrix $R^{(i)}$ as a reconstruction using only one latent component from the approximation matrix, where $i \in\{1, \ldots, r\}$, and $r$ is the factorization rank. $R^{(i)}$ can be seen as a projection on the direction of the $i$-th factor. For example, $R_{\mathrm{STMF}}^{(1)}$ matrix in Fig. $7 \mathrm{a}$ is a result of the $(\max ,+)$ product, which represent sums of each pair of elements, of the first column of $U_{\mathrm{STMF}}$ and the first row of $V_{\mathrm{STMF}}$ (Fig. 6). In the case of NMF, instead of sum, there is multiplication (see Fig. 7b). If we compute an element-wise maximum of all $R_{\mathrm{STMF}}^{(i)}$ we get the $R_{\mathrm{STMF}}$, while element-wise sum of all $R_{\mathrm{NMF}}^{(i)}$ results in $R_{\mathrm{NMF}}$. In this way, we see which latent matrix $R^{(i)}$ explains which part of the data. On the BIC matrix, we see that both methods, STMF, and NMF, describe most of the data with the first latent matrix (Fig. 7). For all other datasets latent matrices are available in Additional file 1, Section 2.

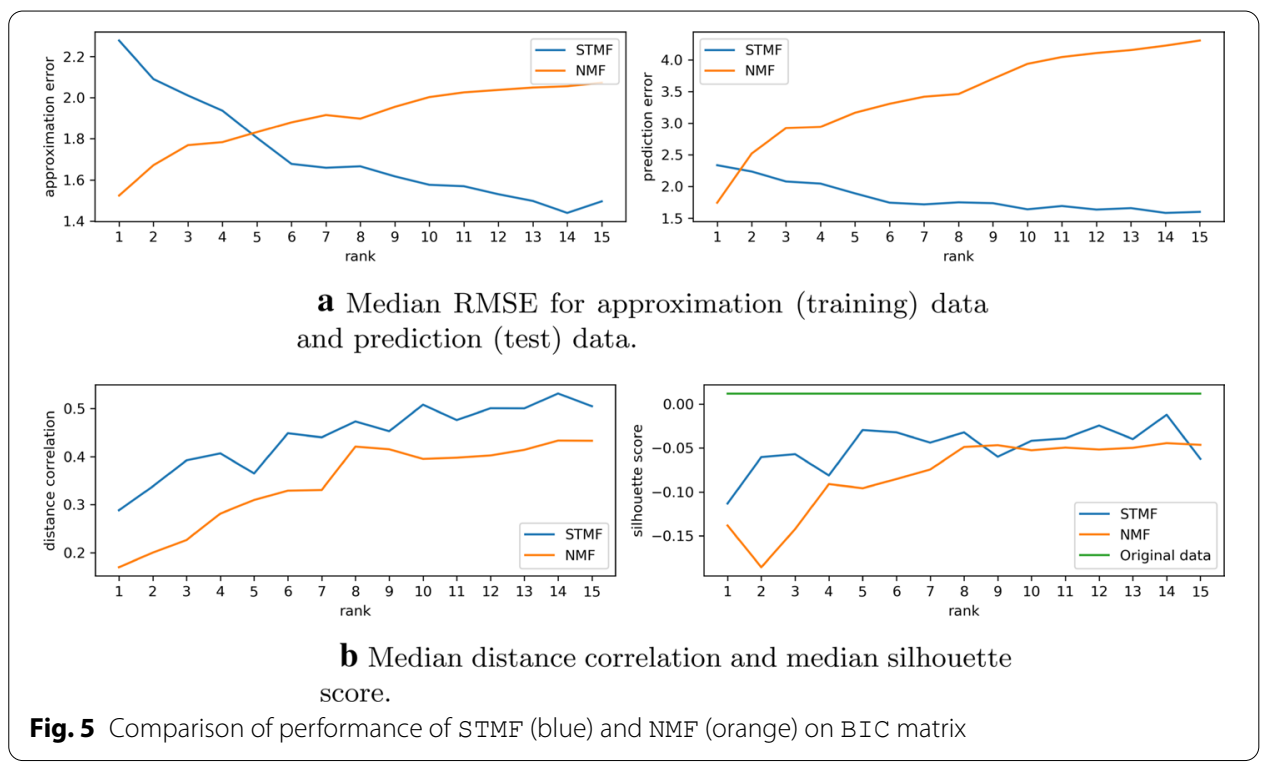




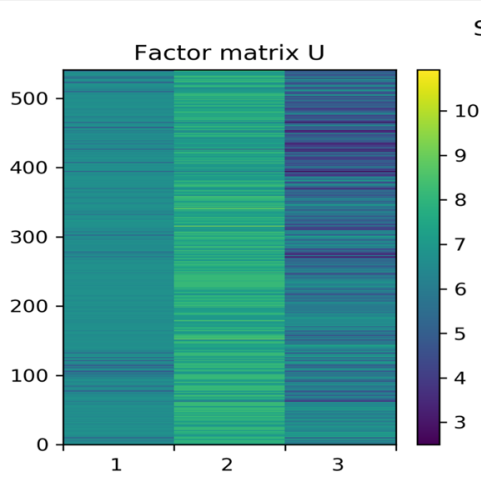

STMF Factor matrix $V$

a Factor matrices $U_{\mathrm{STMF}}, V_{\mathrm{STMF}}$ from STMF.

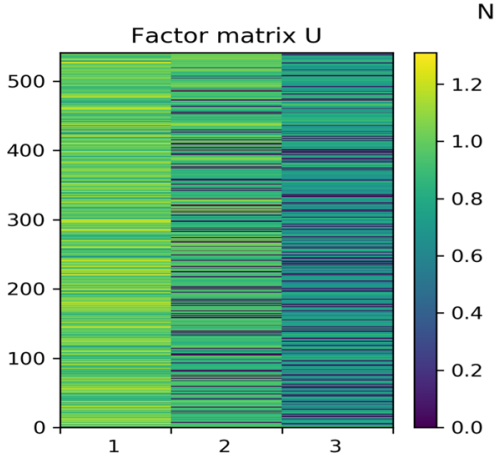

NMF
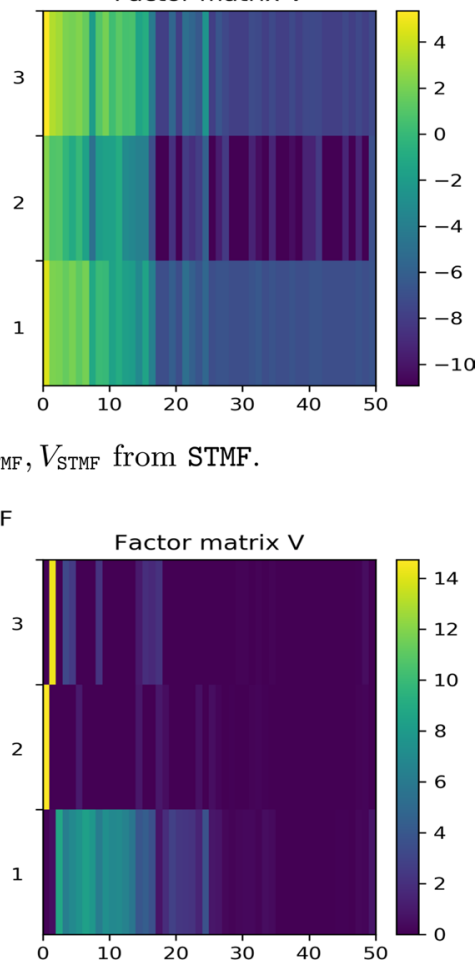

b Factor matrices $U_{\mathrm{NMF}}, V_{\mathrm{NMF}}$ from NMF.

Fig. 6 Factor matrices $U_{S T M F}, V_{S T M F}$ and $U_{\text {NMF }}, V_{N M F}$ obtained by STMF and NMF algorithms, respectively, on BIC matrix

In Table 4 we present the results of experiments on nine datasets listed in Table 2. We see that STMF outperforms NMF on six out of nine datasets, while NMF achieves better results on the LUSC, SKCM and SARC datasets. When we add to the comparison the PMF method, which is the probabilistic version of NMF, it outperforms STMF and NMF on five datasets, but there is no statistically significant difference between the three methods according to the critical difference $(C D)$ method by Demšar [45] (see Additional file 1: Figure S 21).

Solving linear systems using $\otimes^{*}$ emphasizes the low (blue) and high (yellow) gene expression values of patients in Fig. 4. In this way, STMF can, in some cases, recover better the original data, while NMF's results are diluted. However, a limitation of STMF compared to NMF is in its computational efficiency (Table 3).

In Fig. 8 we plot the distribution of Euclidean norm of difference between centered original data and centered approximations of rank $r$ (chosen in Table 4) for different datasets. We see that even if we use another metric like Euclidean norm, computed for each row (patient) separately, results still show that STMF outperforms NMF, as it is shown in Table 4 using distance correlation.

In Fig. 9 we explore the difference between the original, approximated and centered BIC dataset. For every row (patient) we present the Euclidean norm of the difference between the rows in the original and the approximated matrix on $x$-axis, which 


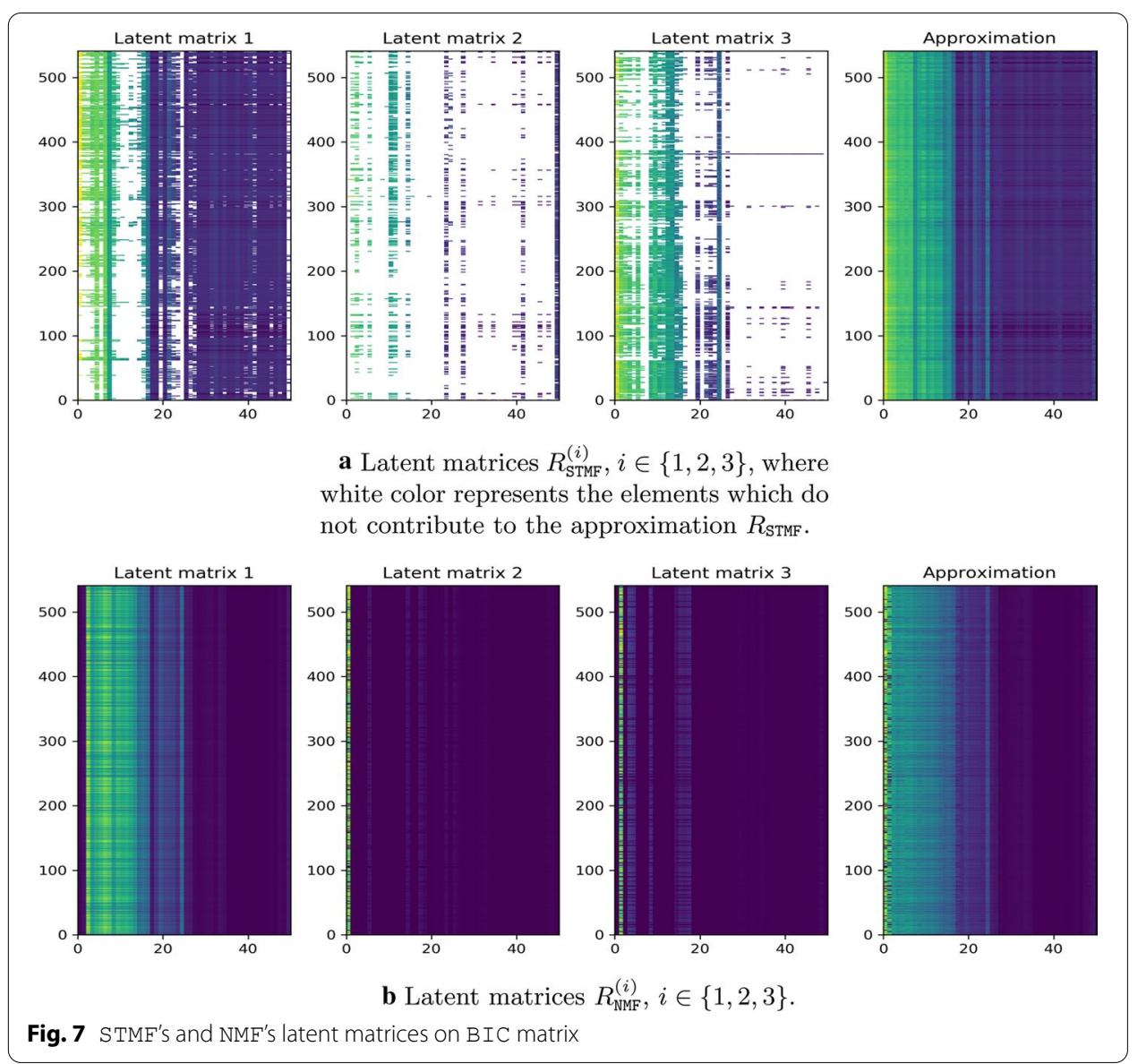

Table 3 Average running times in seconds with the best choice of rank $r$ for different matrix factorization methods on nine datasets

\begin{tabular}{lllll}
\hline Dataset & Rank $\boldsymbol{r}$ & STMF [s] & NMF [s] & PMF [s] \\
\hline AML & 3 & 117.953 & 0.336 & 0.028 \\
COLON & 3 & 153.398 & 0.312 & 0.864 \\
GBM & 3 & 191.204 & 0.353 & 1.996 \\
LIHC & 2 & 236.655 & 0.467 & 1.794 \\
LUSC & 3 & 239.329 & 0.456 & 3.538 \\
OV & 4 & 251.328 & 0.336 & 2.159 \\
SKCM & 3 & 310.401 & 0.395 & 6.309 \\
SARC & 3 & 186.475 & 0.398 & 0.215 \\
BIC & 3 & 221.669 & 0.526 & 0.248 \\
\hline
\end{tabular}

can be interpreted as the accuracy of the approximated values. In contrast, on $y$-axis we present the Euclidean norm of the difference between the corresponding rows in the centered original and centered approximated matrix, which can be interpreted as the average error of the reconstruction of the original pattern. We see that for each row (patient) the STMF's value on $y$-axis is smaller than the NMF's value, indicating that STMF better approximates the original patterns. The rows in the STMF's 
Table 4 Distance correlations with the best choice of rank $r$ for different matrix factorization methods on nine datasets

\begin{tabular}{lllllll}
\hline Dataset & Rank $\boldsymbol{r}$ & \multicolumn{2}{l}{ STMF } & & NMF & \multirow{2}{*}{ PMF } \\
\cline { 3 - 4 } & & Min. & Median & Max. & & \\
\hline AML & 3 & 0.650 & $\mathbf{0 . 8 3 1 ^ { * }}$ & 0.845 & 0.636 & 0.623 \\
COLON & 3 & 0.585 & $\mathbf{0 . 6 4 7}$ & 0.688 & 0.586 & $0.707^{*}$ \\
GBM & 3 & 0.684 & $\mathbf{0 . 7 0 2 ^ { * }}$ & 0.762 & 0.325 & 0.330 \\
LIHC & 2 & 0.493 & $\mathbf{0 . 5 1 5}$ & 0.588 & 0.311 & $0.649^{*}$ \\
LUSC & 3 & 0.498 & 0.562 & 0.731 & $\mathbf{0 . 6 9 7}$ & $0.799^{*}$ \\
OV & 4 & 0.420 & $\mathbf{0 . 5 6 9 ^ { * }}$ & 0.601 & 0.347 & 0.563 \\
SKCM & 3 & 0.480 & 0.521 & 0.605 & $\mathbf{0 . 6 3 3}$ & $0.808^{*}$ \\
SARC & 3 & 0.493 & 0.584 & 0.610 & $\mathbf{0 . 6 4 9 *}$ & 0.588 \\
BIC & 3 & 0.350 & $\mathbf{0 . 3 9 2}$ & 0.531 & 0.227 & $0.427^{*}$
\end{tabular}

Result of best method in the comparison between STMF and NMF shown in bold. Best result among all three methods ( $\mathrm{STMF}, \mathrm{NMF}, \mathrm{PMF}$ ) indicated by asterisk

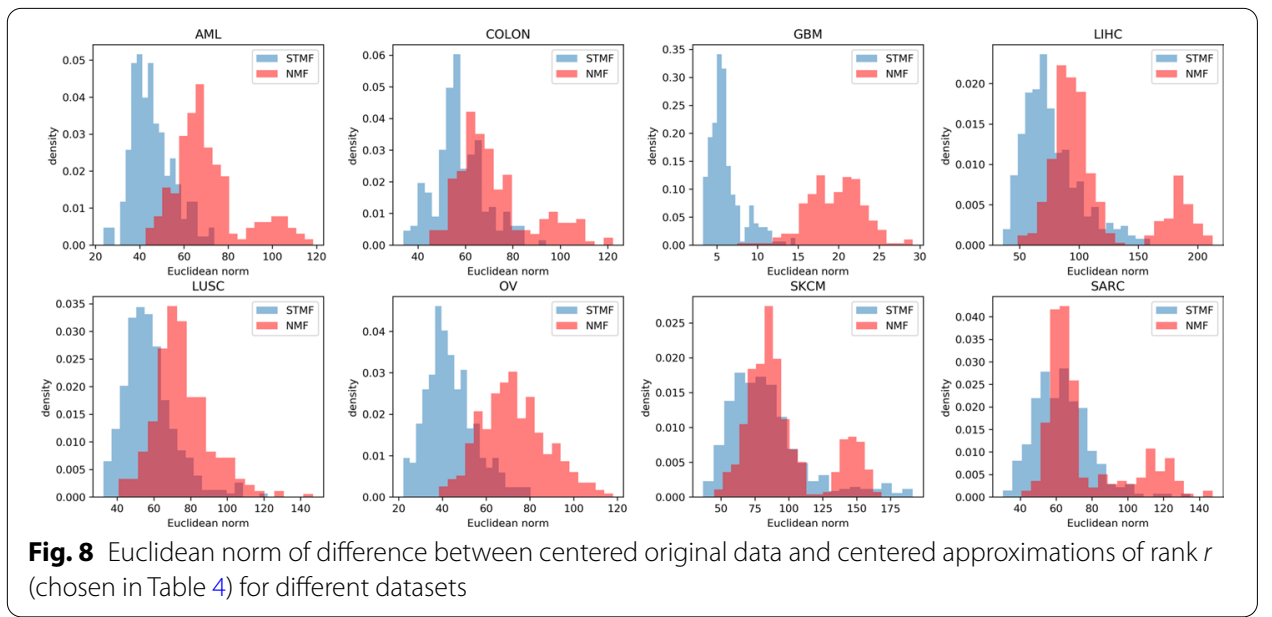

approximation in Fig. 4 with predominantly low values have large approximation errors ( $y$-axis) in Fig. 9 while having a comparable approximation of the original pattern as NMF's approximation of the original pattern.

We see that NMF has two clusters of patients with large values on $y$-axis, denoted by red stars and red circles. These are the rows (patients) where the NMF's predicted pattern differs significantly from the original pattern, more than the STMF's predictions, but at the same time NMF is achieving smaller approximation error than STMF. In Fig. 10 we plot the patients corresponding to these two clusters and compare approximations with original data. It can be seen that NMF cannot model high (yellow) values in a few first columns, while low (blue) values are larger (light blue) compared to the original matrix, which has around half of the data plotted with dark blue. Comparison with Pearson and Spearman correlation is shown in Additional file 1: Figure S 25, where STMF achieves higher Pearson correlation, but lower Spearman correlation. Clusters of patients are also visible in both figures using these two correlations confirming results in Fig. 9. For all other datasets plots are available in Additional file 1, Section 2. 


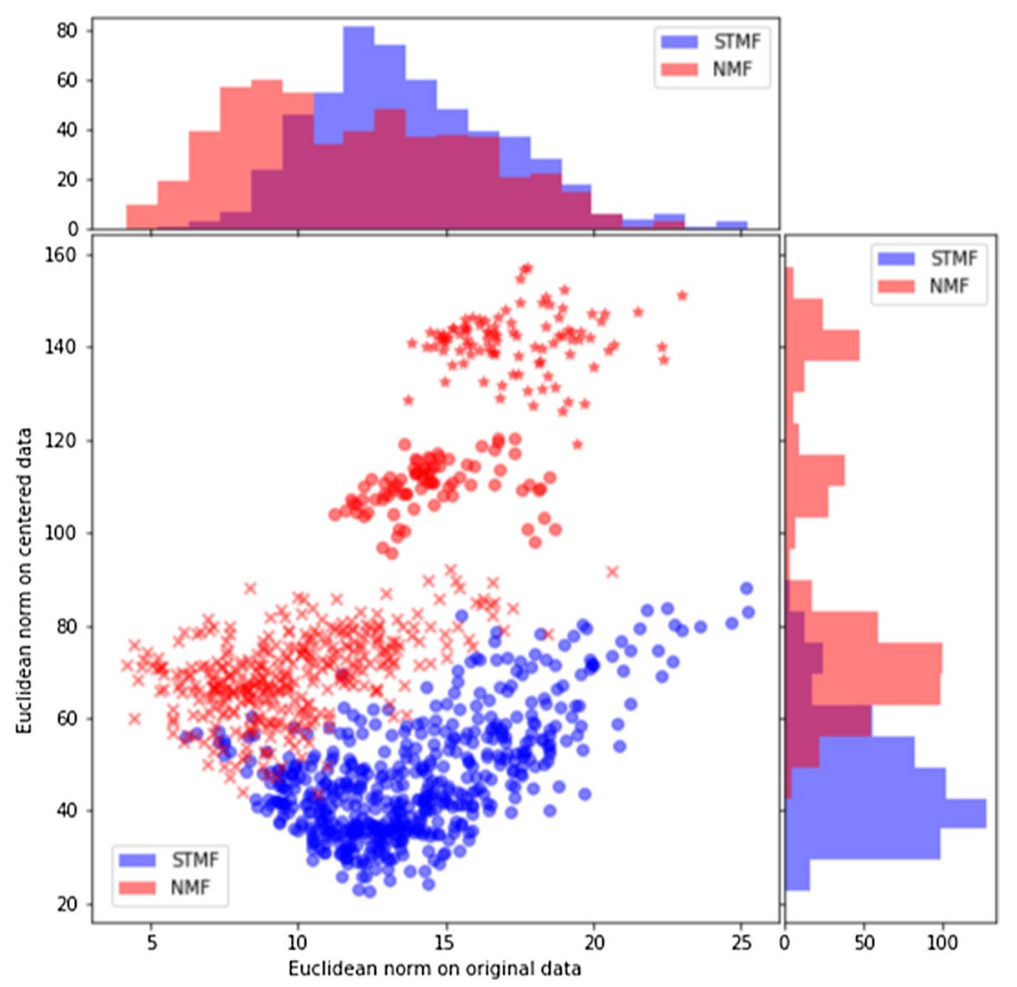

Fig. 9 Euclidean norm of difference between original BIC data and approximations ( $x$-axis) and Euclidean norm of difference between centered original data and centered approximations ( $y$-axis)

\section{Conclusion}

Standard linear algebra is used in the majority of data mining and machine learning tasks. Utilizing different types of semirings has the potential to reveal previously undiscovered patterns. The motivation for using tropical semiring in matrix factorization methods is that resulting factors should give us the most dominant features that are specific and essential for each factor. In that way, factors are likely easier to interpret.

We propose a method called STMF, which can work with missing values. We implement STMF by extending TMF algorithm to be able to handle unknown values. Results show that NMF could not successfully recover the patterns on specific synthetic data, while the approximation with STMF achieves a higher correlation value. Results on TCGA data show that STMF outperforms NMF in the prediction task. Also, the results obtained by NMF tend toward the mean value, while the approximations obtained by STMF better express extreme values. Our proposed approach identifies strong patterns that aid the visual interpretation of results. In this way, we can discover sharp, high-variance components in the data. To the best of our knowledge, STMF is the first work using tropical semiring in sparse (biomedical) data.

A limitation of our STMF method is its apparent inability to embed and predict truly novel examples (i.e., new incomplete rows or columns in the data matrix). Developing an approach similar to the one we have shown for NMF [17] deserves further research to address this important task. 


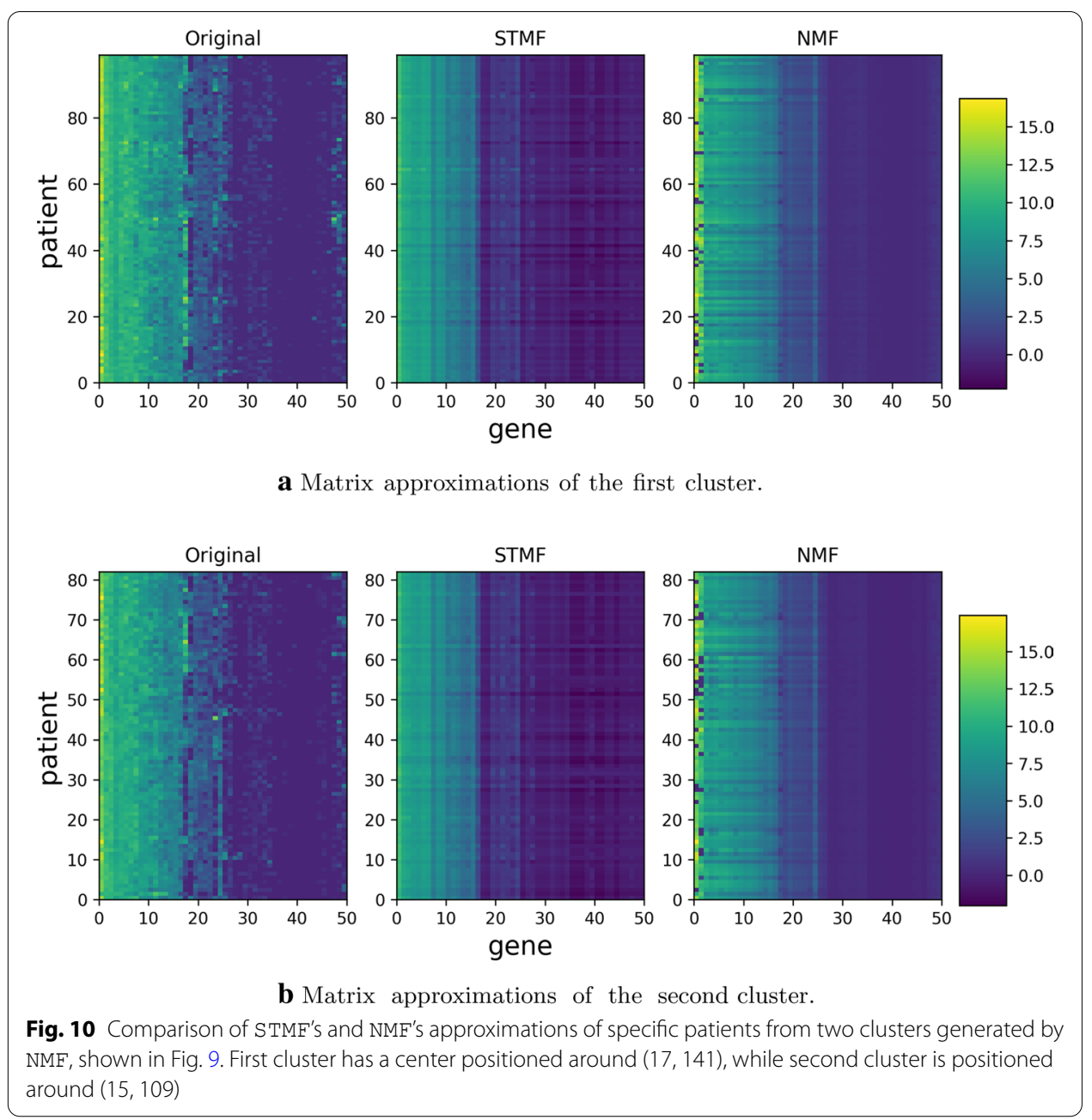

Another limitation of STMF method is the fact that can be used only on single data source. Integrative data fusion methods are based on co-factorization of multiple data matrices. Using standard linear algebra, $D F M F$ is a variant of penalized matrix trifactorization, which simultaneously factorizes data matrices to reveal hidden associations. It can model multiple relations between multiple object types, while relations between some object types can be completely missing. In our future work, we will investigate ways to modify the STMF method for data fusion of multiple data sources focusing on the fusion of methylation, miRNA, and gene expression data.

We believe that future research will show that semirings are useful in many scenarios and that they find the structure that is different and easier to interpret than with standard linear algebra.

\section{Supplementary Information}

The online version contains supplementary material available at https://doi.org/10.1186/s12859-021-04023-9. 


\section{Acknowledgements}

We would like to express very great appreciation to Rafsan Ahmed from Antalya Bilim University (Department of Computer Engineering, Machine Learning Laboratory, now at Lund University), who helped us acquire and preprocess the real data.

The results published here are in whole or part based upon data generated by the TCGA Research Network: https:// www.cancer.gov/tcga.

\section{Authors' contributions}

$\mathrm{AO}, \mathrm{TC}$ and $\mathrm{PO}$ designed the study. HK guided the selection and the processing of the data. AO wrote the software application and performed experiments. $\mathrm{AO}$ and TC analyzed and interpreted the results on real data. AO wrote the initial draft of the paper. All authors read and approved the final manuscript.

\section{Funding}

This work is supported by the Slovene Research Agency, Young Researcher Grant (52096) awarded to AO, and research core funding (P1-0222 to PO and P2-0209 to TC).

\section{Availability of data and materials}

This paper uses the real TCGA data available on http://acgt.cs.tau.ac.il/multi_omic_benchmark/download.html. PAM50 data can be found on the https://github.com/CSB-IG/pa3bc/tree/master/bioclassifier_R/. BIC subtypes are collected from https://www.cbioportal.org/. STMF code, PAM50 data and BIC subtypes are available on https://github.com/Ejmri C/STMF.

\section{Ethics approval and consent to participate}

Not applicable.

\section{Consent for publication}

Not applicable.

\section{Competing interests}

The authors declare that they have no competing interests.

\section{Author details}

${ }^{1}$ Faculty of Computer and Information Science, University of Ljubljana, Večna pot 113, 1000 Ljubljana, Slovenia. ${ }^{2}$ Department of Computer Engineering, Antalya Bilim University, Çıplakl, Akdeniz Blv. No:290/A, 07190 Antalya, Turkey.

Received: 27 November 2020 Accepted: 8 February 2021

Published online: 25 February 2021

\section{References}

1. Koren Y, Bell R, Volinsky C. Matrix factorization techniques for recommender systems. Computer. 2009;42(8):30-7.

2. Xu W, Liu X, Gong Y. Document clustering based on non-negative matrix factorization. In: Proceedings of the 26th annual international ACM SIGIR conference on research and development in information retrieval, 2003. p. 267-273

3. Brunet J-P, Tamayo P, Golub TR, Mesirov JP. Metagenes and molecular pattern discovery using matrix factorization. Proc Nat Acad Sci. 2004:101(12):4164-9.

4. Karaev S, Hook J, Miettinen P. Latitude: a model for mixed linear-tropical matrix factorization. In: Proceedings of the 2018 SIAM international conference on data mining, 2018. p. 360-368. SIAM.

5. Lee DD, Seung HS. Learning the parts of objects by non-negative matrix factorization. Nature. 1999;401 (6755):788

6. Koren Y, Bell R, Volinsky C. Matrix factorization techniques for recommender systems. Computer. 2009;8:30-7.

7. Zhang Z, Li T, Ding C, Zhang X. Binary matrix factorization with applications. In: Seventh IEEE international conference on data mining (ICDM 2007), 2007. p. 391-400. IEEE.

8. Zhang Z-Y, Li T, Ding C, Ren X-W, Zhang X-S. Binary matrix factorization for analyzing gene expression data. Data Min Knowl Disc. 2010;20(1):28.

9. Mnih A, Salakhutdinov RR. Probabilistic matrix factorization. In: Advances in neural information processing systems, 2008. p. 1257-1264.

10. Wang JJ-Y, Gao X. Max-min distance nonnegative matrix factorization. Neural Netw. 2015;61:75-84.

11. Rappoport N, Shamir R. Multi-omic and multi-view clustering algorithms: review and cancer benchmark. Nucleic Acids Res. 2018:46(20):10546-62

12. Liu J, Wang C, Gao J, Han J. Multi-view clustering via joint nonnegative matrix factorization. In: Proceedings of the 2013 SIAM international conference on data mining, 2013. p. 252-260. SIAM.

13. Zhang S, Liu C-C, LiW, Shen H, Laird PW, Zhou XJ. Discovery of multi-dimensional modules by integrative analysis of cancer genomic data. Nucleic Acids Res. 2012;40(19):9379-91.

14. Li S-Y, Jiang Y, Zhou Z-H. Partial multi-view clustering. In: Twenty-eighth AAAl conference on artificial intelligence 2014.

15. Žitnik M, Zupan B. Data fusion by matrix factorization. IEEE Trans Pattern Anal Mach Intell. 2015;37(1):41-53.

16. Wang JJ-Y, Gao X. Beyond cross-domain learning: multiple-domain nonnegative matrix factorization. Eng Appl Artif Intell. 2014;28:181-9.

17. Stražar M, Žitnik M, Zupan B, Ule J, Curk T. Orthogonal matrix factorization enables integrative analysis of multiple RNA binding proteins. Bioinformatics. 2016:32(10):1527-35.

18. Karaev S, Miettinen P. Algorithms for approximate subtropical matrix factorization. arXiv preprint arXiv:1707.08872. 2017. 
19. Karaev S, Miettinen P. Capricorn: an algorithm for subtropical matrix factorization. In: Proceedings of the 2016 SIAM international conference on data mining, 2016. p. 702-710. SIAM.

20. Karaev S, Miettinen P. Cancer: another algorithm for subtropical matrix factorization. In: Joint European conference on machine learning and knowledge discovery in databases, 2016. p. 576-592. Springer.

21. Weston J, Weiss RJ, Yee H. Nonlinear latent factorization by embedding multiple user interests. In: Proceedings of the 7th ACM conference on recommender systems, 2013. p. 65-68.

22. Le Van T, Nijssen S, Van Leeuwen M, De Raedt L. Semiring rank matrix factorization. IEEE Trans Knowl Data Eng. 2017;29(8):1737-50.

23. De Schutter B, De Moor B. Matrix factorization and minimal state space realization in the max-plus algebra. In: Proceedings of the 1997 American control conference (Cat. No. 97CH36041), 1997, vol. 5, pp. 3136-3140. IEEE.

24. Baccelli F, Cohen G, Olsder GJ, Quadrat J-P. Synchronization and linearity: an algebra for discrete event systems 1992

25. Heidergott B, Olsder GJ, Van Der Woude J. Max plus at work: modeling and analysis of synchronized systems: a course on max-plus algebra and its applications, vol. 48. Princeton: Princeton University Press; 2014.

26. Hook J. Linear regression over the max-plus semiring: algorithms and applications. arXiv preprint arXiv:1712.03499. 2017.

27. Gärtner B, Jaggi M. Tropical support vector machines. Technical report, Technical Report ACS-TR-362502-01 2008.

28. Zhang L, Naitzat G, Lim L-H. Tropical geometry of deep neural networks. arXiv preprint arXiv:1805.07091. 2018.

29. Han P, Yang P, Zhao P, Shang S, Liu Y, Zhou J, Gao X, Kalnis P. GCN-MF: Disease-gene association identification by graph convolutional networks and matrix factorization. In: Proceedings of the 25th ACM SIGKDD international conference on knowledge discovery \& data mining, 2019;705-713.

30. Declerck P, Alaoui MKD. Optimal control synthesis of timed event graphs with interval model specifications. IEEE Trans Autom Control. 2009:55(2):518-23.

31. Akian M, Bapat R, Gaubert S. Asymptotics of the perron eigenvalue and eigenvector using max-algebra. Comptes Rendus de l'Académie des Sciences-Series I-Mathematics. 1998;327(11):927-32.

32. Gunawardena J. Min-max functions. Discrete Event Dyn Syst. 1994;4(4):377-407.

33. Leung H. Limitedness theorem on finite automata with distance functions: an algebraic proof. Theoret Comput Sci. 1991;81(1):137-45.

34. Gaubert S, Plus M. Methods and applications of $(\max ,+)$ linear algebra. In: Annual symposium on theoretical aspects of computer science, 1997;261-282. Springer.

35. Langville AN. Meyer CD, Albright R, Cox J, Duling D. Algorithms, initializations, and convergence for the nonnegative matrix factorization. arXiv preprint arXiv:1407.7299. 2014

36. Clark M. A comparison of correlation measures. Center for Social Research, University of Notre Dame 4, 2013.

37. Székely GJ, Rizzo ML, et al. Brownian distance covariance. Ann Appl Stat. 2009;3(4):1236-65.

38. Bar-Joseph Z, Gifford DK, Jaakkola TS. Fast optimal leaf ordering for hierarchical clustering. Bioinformatics. 2001;17(Suppl_1):22-9.

39. Murtagh F, Legendre P. Ward's hierarchical agglomerative clustering method: which algorithms implement ward's criterion? J Classif. 2014;31(3):274-95.

40. Parker JS, Mullins M, Cheang MC, Leung S, Voduc D, Vickery T, Davies S, Fauron C, He X, Hu Z, et al. Supervised risk predictor of breast cancer based on intrinsic subtypes. J Clin Oncol. 2009;27(8):1160.

41. Cerami E, Gao J, Dogrusoz U, Gross BE, Sumer SO, Aksoy BA, Jacobsen A, Byrne CJ, Heuer ML, Larsson E, et al. The cBio cancer genomics portal: an open platform for exploring multidimensional cancer genomics data. AACR 2012.

42. Gao J, Aksoy BA, Dogrusoz U, Dresdner G, Gross B, Sumer SO, Sun Y, Jacobsen A, Sinha R, Larsson E, et al. Integrative analysis of complex cancer genomics and clinical profiles using the cBioPortal. Sci Signal. 2013;6(269):1.

43. Rousseeuw PJ. Silhouettes: a graphical aid to the interpretation and validation of cluster analysis. J Comput Appl Math. 1987;20:53-65. https://doi.org/10.1016/0377-0427(87)90125-7.

44. Lin X, Boutros PC. Optimization and expansion of non-negative matrix factorization. BMC Bioinform. 2020;21(1):1-10.

45. Demšar J. Statistical comparisons of classifiers over multiple data sets. J Mach Learn Res. 2006;7(Jan):1-30.

\section{Publisher's Note}

Springer Nature remains neutral with regard to jurisdictional claims in published maps and institutional affiliations.

Ready to submit your research? Choose BMC and benefit from:

- fast, convenient online submission

- thorough peer review by experienced researchers in your field

- rapid publication on acceptance

- support for research data, including large and complex data types

- gold Open Access which fosters wider collaboration and increased citations

- maximum visibility for your research: over $100 \mathrm{M}$ website views per year

At BMC, research is always in progress.

Learn more biomedcentral.com/submissions 